OPEN ACCESS

Edited by:

Carryl L. Baldwin,

George Mason University, USA

Reviewed by:

Ramesh Kandimalla,

Texas Tech University, USA

Neha Sehgal,

Wisconsin Institute for Discovery, USA

${ }^{*}$ Correspondence:

Tiffany Tong

tiffany.tong@mail.utoronto.ca

Received: 17 June 2016 Accepted: 18 October 2016 Published: 07 November 2016

Citation:

Tong T, Chignell M, Tierney MC and Lee JS (2016) Test-Retest Reliability of a Serious Game for Delirium

Screening in the Emergency

Department.

Front. Aging Neurosci. 8:258. doi: 10.3389/fnagi.2016.00258

\section{Test-Retest Reliability of a Serious Game for Delirium Screening in the Emergency Department}

\author{
Tiffany Tong ${ }^{1,2 *}$, Mark Chignell ${ }^{1,2}$, Mary C. Tierney ${ }^{3,4}$ and Jacques S. Lee ${ }^{5}$ \\ 1 Interactive Media Lab, Department of Mechanical and Industrial Engineering, University of Toronto, Toronto, ON, Canada, \\ ${ }^{2}$ Knowledge Media Design Institute, Faculty of Information, University of Toronto, Toronto, ON, Canada, ${ }^{3}$ Department of \\ Family and Community Medicine, University of Toronto, Toronto, ON, Canada, ${ }^{4}$ Primary Care Research Unit, Sunnybrook \\ Health Sciences Centre, Toronto, ON, Canada, ${ }^{5}$ Clinical Epidemiology Unit, Department of Emergency Services, \\ Sunnybrook Health Sciences Center, Toronto, ON, Canada
}

Introduction: Cognitive screening in settings such as emergency departments (ED) is frequently carried out using paper-and-pencil tests that require administration by trained staff. These assessments often compete with other clinical duties and thus may not be routinely administered in these busy settings. Literature has shown that the presence of cognitive impairments such as dementia and delirium are often missed in older ED patients. Failure to recognize delirium can have devastating consequences including increased mortality (Kakuma et al., 2003). Given the demands on emergency staff, an automated cognitive test to screen for delirium onset could be a valuable tool to support delirium prevention and management. In earlier research we examined the concurrent validity of a serious game, and carried out an initial assessment of its potential as a delirium screening tool (Tong et al., 2016). In this paper, we examine the test-retest reliability of the game, as it is an important criterion in a cognitive test for detecting risk of delirium onset.

Objective: To demonstrate the test-retest reliability of the screening tool over time in a clinical sample of older emergency patients. A secondary objective is to assess whether there are practice effects that might make game performance unstable over repeated presentations.

Materials and Methods: Adults over the age of 70 were recruited from a hospital ED. Each patient played our serious game in an initial session soon after they arrived in the ED, and in follow up sessions conducted at 8-h intervals (for each participant there were up to five follow up sessions, depending on how long the person stayed in the ED).

Results: A total of 114 adults (61 females, 53 males) between the ages of 70 and 104 years $(M=81$ years, $S D=7)$ participated in our study after screening out delirious patients. We observed a test-retest reliability of the serious game (as assessed by correlation $r$-values) between 0.5 and 0.8 across adjacent sessions.

Conclusion: The game-based assessment for cognitive screening has relatively strong test-retest reliability and little evidence of practice effects among elderly emergency patients, and may be a useful supplement to existing cognitive assessment methods.

Keywords: cognitive screening, human factors, serious games, delirium, gerontology, test-retest reliability 


\section{INTRODUCTION}

In many countries, aging populations place serious demands on healthcare systems. The cost of healthcare spending per adult aged 65 years and older has been estimated to be three to five times more than the corresponding cost for younger individuals (Glass and Balfour, 2003; Centers for Disease Control and Prevention, 2013). The rise in healthcare expenditures due to agerelated conditions has prompted research on how to minimize costs while maximizing care for adults through early screening, monitoring and intervention methods (Zaslavsky et al., 2012). Cognitive screening of older people is important to monitor chronic (e.g., dementia) or acute (e.g., delirium) changes in cognitive status. In the chronic context, cognitive assessment is needed to monitor risk of dementia for people likely to have cognitive impairment (Petersen et al., 2001). In both primary care and the ED, recognition of cognitive impairment has repeatedly been shown to be poor. In primary care, (Tierney and Lermer, 2010): "the available data indicate that the substantial rates of under-utilization of existing cognitive tools in primary care are mainly due to lack of time, lack of training, lack of tools perceived as helpful, and lack of confidence."

Cognitive screening is particularly important in the case of elderly patients at risk for delirium. Delirium is a serious, and potentially fatal problem affecting up to $50 \%$ of hospitalized seniors, and costing over $\$ 164$ billion per year in the US as of 2011 (Inouye et al., 2014). Failure to detect delirium is associated with poorer outcomes, including a two-to-three fold increase in mortality (Kakuma et al., 2003; Pun and Ely, 2007). However detection can be improved by routine cognitive testing (Meagher et al., 2001). Patients with intensive care unit delirium have more than a threefold-increased risk of 6-month mortality compared to those without delirium.

Since one of the key properties of delirium is a fluctuating course (Inouye, 1990; Meagher et al., 2001), where a patient may appear normal at one point, and show signs of confusion hours later, repeated cognitive assessment is needed to assess both the onset, and risk of onset, of delirium.

Risk of delirium is also elevated for elderly patients undergoing surgery. Rudra et al. (2006) argued that "good preoperative evaluation should include a formal cognitive assessment in patients at risk of developing delirium." In longterm care, prevalence of delirium has been reported to range between 1.4 and $70 \%$, depending on diagnostic criteria and on the prevalence of dementia (de Lange et al., 2013). Risk of delirium is particularly high for people with dementia, who are over the age of 85, or who are living in a care facility (de Lange et al., 2013). Delirium has been estimated to be present in 7 to $10 \%$ of older patients in the ED (Hustey et al., 2000; Hustey and Meldon, 2002; LaMantia et al., 2014). However, emergency providers identify delirious patients in only 16 to $35 \%$ of cases (Hustey et al., 2000).

Abbreviations: CAM, Confusion Assessment Method; CANTAB, Cambridge Automated Neuropsychological Testing Battery; CRT, Choice Reaction Time; DI, Delirium Index; DVT, Digit Vigilance Task; ED, Emergency Department; M, Mean; MMSE, Mini Mental State Exam; MoCA, Montreal Cognitive Assessment; RA, Research Assistant; RASS, Richmond Agitation-Sedation Scale; RT, Response Time; SD, Standard Deviation; SE, Standard Error.
Thus there is a clear need for better screening of delirium in the ED, not only when elderly patients are admitted, but also during their sometimes lengthy stays in the ED where they may transition to a delirious state.

Earlier, we developed a tablet-based serious game for cognitive assessment (Tong and Chignell, 2014). Tong et al. (2016) carried out an initial concurrent validation (with existing methods of clinical assessment) of the game in an ED, finding significant correlations of game performance with scores on the MMSE (Folstein et al., 1975) and the MoCA (Nasreddine et al., 2005). Tong et al. (2016) also found evidence that the serious game may be useful as an initial screen for delirium, with game RT differing significantly between CAM (Inouye et al., 1990) positive and CAM negative patients in a sample of elderly emergency patients. In this paper we examine the test-retest reliability (e.g., Anastasi, 1988) of our serious game as a further investigation of its psychometric properties, with the motivating application being screening for delirium in an ED.

\section{BACKGROUND INFORMATION}

\section{Cognitive Assessment}

The most frequently used clinical tests of cognitive ability in the elderly, such as the MMSE and MoCA, are pencil-andpaper based. The MMSE and MoCA have high test-retest reliabilities, reported between 0.80 and 0.95 for the MMSE (Tombaugh, 2005), and 0.92 for the MoCA (Nasreddine et al., 2005). These assessments are administered by having trained personnel ask patients for verbal or written responses. Thus, they are not designed or suitable for self-assessment in nonclinical environments. They can also be time consuming to carry out in a busy clinical setting such as an ED.

Challenges associated with current methods of cognitive assessment include limited alternate versions of paper-based tests, which can subsequently lead to practice effects. Practice effects have been shown with the MMSE in both healthy adults and those diagnosed with dementia at short test-retest intervals ranging from 10 min to 1.5 weeks (Galasko et al., 1993; JacqminGadda et al., 1997), and longer intervals of three months (Helkala et al., 2002). Moreover, the MoCA has also demonstrated practice effects in longitudinal performance of healthy older adults (Cooley et al., 2015). The presence of practice effects in cognitive assessments is potentially due to a limited range of questions, and these effects are most prominent on questions evaluating the domains of visual memory, attention, working memory, processing speed, and executive functioning (Cooley et al., 2015). A meta-analysis by Calamia et al. (2012) revealed that practice effects are more pronounced with short test-retest intervals.

Some existing cognitive screening tools have been modified to increase their accessibility and use for different types of patients. For example, the MoCA has an alternative-scoring schema for patients with only a high-school level education (Nasreddine et al., 2005), and the CAM has also been adapted for use in intensive care units (Ely et al., 2001). However, tests tend to be limited in terms of when and how they can be used. For instance, paper-and-pencil tests that require written input (such as the 
clock drawing task component on the MoCA) may be difficult to complete for patients with limited ranges of motion or other physical disabilities, or for the bed bound.

\section{Electronic Cognitive Assessment}

With the availability of the Internet, and electronic devices such as tablets and smartphones, there has been a shift towards designing electronic cognitive assessments, and translating existing screening tools into a digital medium. The use of technology can provide many benefits, including the ability to record information such as RT and accuracy with precision (Collerton et al., 2007; Wild et al., 2008). Data collection using technology can assist in reducing errors in transcribing paperbased results into digital formats. Once collected, electronic cognitive assessments can also be easily shared between healthcare professionals and patients.

Existing computer-based software for cognitive assessment includes the CANTAB (Cambridge Cognition Ltd, 2014), CNS Vital Signs (Gualtieri and Johnson, 2006), and CAMCI (Saxton et al., 2009). Aside from computer-based testing, other form factors such as smartphones and touch-based tablets have been explored in tools such as the CADi, which screens for dementia (Onoda et al., 2013), the CST, which screens for general cognitive function (Brouillette et al., 2013), and DETECT, which screens for MCI in elderly patients in primary care settings (Wright et al., 2010). The National Institutes of Health (2012) has introduced the NIH Toolbox, which contains an array of tests that monitor neurological and behavioral function (Gershon et al., 2013). The NIH Toolbox requires a test administrator to run and score the tests, which cannot be self-administered by patients.

\section{Serious Games for Cognitive Assessment}

There has been growing interest in the use of serious games to assess cognitive status. Serious games are games designed with a primary purpose other than entertainment (Charsky, 2010). Examples include the ElderGAMES Project (Gamberini et al., 2006), which uses a tabletop setup designed for use by multiple users. Other work by Anguera et al. (2013) has explored the use of driving simulation as a means for brain training and assessment. The use of virtual reality devices has also been explored as a method to assess cognitive function with serious games as exemplified by the work of (Zuchella et al., 2014). Current gamebased approaches to cognitive screening are limited by lack of validation with clinical populations and insufficient reliability testing.

In the remainder of this paper we report on a study that assessed the test-retest reliability of a serious game for cognitive assessment, within an ED. One benchmark for comparing the test-retest reliability of the serious game is the test-retest reliability results for the MMSE. Test-retest reliability assessments for the MMSE typically used cognitively intact individuals and test-retest intervals of less than 6 months. The reliability estimates generally fell between 0.80 and 0.95 (Tombaugh and McIntyre, 1992). Tombaugh (2005) examined test-retest reliabilities of the MMSE for people without cognitive impairment over different pairs of four time periods that varied between 1 and 5 years apart. The test-retest reliabilities over these longer time periods were lower, varying between 0.48 and 0.65 .

\section{MATERIALS AND METHODS}

\section{Procedure}

We conducted an observational cohort study between January, 2015 and October, 2015. Our protocol for the study was approved by institutional review boards at the Sunnybrook Health Sciences Centre and the University of Toronto (protocols 070-2013 and 28953, respectively). The study was carried out in accordance with the recommendations of both review ethics boards with written informed consent obtained from all participants.

Trained clinical RAs administered the following standard cognitive assessments during the initial enrolment: MMSE, MoCA, DI (McCusker et al., 2004), RASS (Sessler et al., 2002), a DVT (Kelland, 1996), and a CRT task. Following this, the RA asked patients to play the serious game-based assessment.

During follow up sessions, RAs administered the MMSE, DI, CAM, DVT, and the serious game.

The serious game was a tablet-based version of a go, nogo discrimination task (Yechiam et al., 2006) in the form of a whack-a-mole game (Tong and Chignell, 2014), and there were two primary performance measures: RT and target offset. RT was measured as the time between the appearance of a target and user's response, and target offset was measured as the pixel distance between the center of the target and the center of the user's touch.

\section{Patient Selection}

Potential subjects were screened using the ED Information System at Sunnybrook Hospital part of the Sunnybrook Health Sciences Centre in Ontario, Canada. We approached patients who presented during study hours, regardless of their presenting state. The inclusion criteria for potential subjects included (1) being 70 years of age or older, and (2) present in the ED for a minimum of 8 -h.

The exclusion criteria for subjects included: (1) being critically ill (defined by a Canadian Triage Acuity Scale score of 1 , (2) having acute pain (a Numeric Rating Scale $\geq 2 / 10$ ), (3) currently receiving psychoactive medications, (4) having a psychiatric primary presenting complaint, (5) having been previously enrolled, or (6) not speaking English or being unable to follow commands or communicate verbally and (7) having hand injuries preventing use of the tablets. We also screened patients with the CAM and removed patients from the study if they were found to be CAM positive in the initial session, or in any of the follow up sessions. Since patients with delirium will typically have fluctuating cognitive status, they were not considered in assessing test-retest reliability.

\section{Test-Retest Reliability}

The test-retest reliability of the serious game was assessed by conducting follow-ups at regular time intervals a minimum of 8-h 
apart, on CAM negative patients. Patients varied in the number of follow-ups they participated in, depending on their total time in the ED. During each follow up, the RA administered the MMSE, DI, CAM, and DVT, and asked the patient to play the serious game. For each patient, there was a maximum of five followup sessions, in addition to the initial enrolment into the study. Reliability of game scores between pairs of sessions was tested using both Pearson and Spearman correlations.

\section{Statistical Analysis}

The assumption of normality was checked for the MMSE scores, as well as for the serious game median RT and target offset data. Data normality was visually inspected using histograms, $\mathrm{P}-\mathrm{P}$ and $\mathrm{Q}-\mathrm{Q}$ plots. Due to the large sample size, tests such as the Kolmogorov-Smirnov and Shapiro-Wilk were not carried out as they are overly sensitive with large sample sizes (Field, 2013). Results from the MMSE were treated as interval data. The median RT data for the serious game was positively skewed and no data transformations were performed. However, the target offset data were normally distributed. Median RTs were used to summarize the RT data in order to reduce the impact of positive skew and outliers on analyses with the RT data. In addition, nonparametric tests were also used as an alternative interpretation of the data without making normality assumptions (Spearman's rho, and the Wilcoxon signed-rank test).

\section{RESULTS}

\section{Study Sample}

A total of 114 patients participated in the study, between the ages of 70 and 104 years $(S D=7)$. There were 61 females, and 53 males in the sample. The average length of stay in the ED was $16.3 \mathrm{~h}$ $(S D=9.0)$ (Table 1).

\section{Completion Rate}

Of the 114 participants who played the serious game in the initial session, 47, 23, and 16 patients (who were assessed as CAM negative played the game in follow up sessions 1, 2, and 3, respectively. Of this set, the numbers of people also completing other assessments, during the initial session and the follow up sessions, is shown in Table 2.

\section{Cognitive Assessment Demographics}

At initial enrolment, MMSE scores ranged from 12 to 30, and MoCA scores ranged from 10 to 30 . The ranges and distribution of scores for each cognitive assessment based on each session are displayed in Table 2. The MoCA, RASS, and CRT were only

TABLE 1 | Demographics of the study sample.

Baseline features

Mean age (years) $(S D)$

Female $(n)$

Male (n)

Mean length of stay in the ED (hours) (SD)
$81.1(7.0)$

61

53

$16.3(9.0)$ carried out during initial enrolment and thus their test-retest reliability was not assessed. Some MMSE assessment and game performance data was missing for participants who either refused to complete the assessment, or were fatigued or sleeping when RAs came to assess them.

\section{Test-Retest Reliability}

The test-retest reliability of the serious game was investigated by calculating two-tailed Pearson's $r$ correlations between pairs of sessions (Table 3 shows correlations for median RT and Table 4 shows correlations for median target offsets). While Table 3 shows all possible pairwise correlations, the three correlations between adjacent time periods are shaded and each correlation in these shaded time periods was significant $(p<0.05)$ with $r$-values ranging between 0.56 and 0.82 . Corresponding scatterplots for the three adjacent pairs of sessions are shown in Figure $\mathbf{1}$. A similar correlation analysis was carried out using the serious game median target offset values (Table 4), with corresponding scatterplots between adjacent time periods being shown in Figure 2. All adjacent pairs of sessions had strong correlations, with $r$-values varying between 0.49 and 0.80 .

The test-retest reliability of the serious game median RT and median target offset were recalculated using Spearman's rho correlations (Tables 5 and 6) at each follow up. The rho-values were all significant $(p<0.05)$ across the three adjacent follow-up sessions for both RT (rho-values ranging between 0.56 and 0.85 ) and target offset (rho-values between 0.68 and 0.77 ).

\section{Practice and Fatigue Effects}

We carried out inferential tests to assess the statistical significance of possible practice/learning effects. Three paired $t$-tests (twotailed) were carried out to determine if there was a difference in game median RT between (1) initial enrolment and follow up 1, (2) follow up sessions 1 and 2, and (3) follow up sessions 2 and 3. Bar charts corresponding to these comparisons are shown in Figure 3 where it can be seen that there is a decreasing trend in median RT across the sessions.

There was no significant difference between the initial enrolment and first follow up session or between the second and third follow up sessions. However, patients had a significantly greater game median RT in the first follow up session $(M=0.9$, $S E=0.06)$ than in the second follow up session $(M=0.8$, $S E=0.03), t(18)=2.384, p=0.028, r=0.821$. In contrast, the corresponding Wilcoxon signed-rank tests indicated that all pairs of adjacent sessions were significant: (1) initial enrolment and follow up session $1(Z=-2.374, p=0.018)$, (2) follow up sessions 1 and $2(Z=-2.696, p=0.007)$, and (3) follow up sessions 2 and $3(Z=-2.103, p=0.035)$.

Paired samples $t$-tests were also conducted using the game target offset values for patients to examine the difference in performance between adjacent sessions (i.e., between the initial session and follow up 1, between follow up sessions 1 and 2, and between follow up sessions 2 and 3). On average, patients were significantly less accurate in the initial enrolment $(M=331.9, S E=5.6)$ versus follow up session 1 $(M=310.5 S E=6.1), t(47)=5.050, p=0.000, r=0.743$ (Figure 4). However, significant differences in game target 
TABLE 2 | Distribution of cognitive assessment scores and game performance.

\begin{tabular}{|c|c|c|c|c|c|c|}
\hline Assessment & Initial enrolment & Follow up 1 & Follow up 2 & Follow up 3 & Follow up 4 & Follow up 5 \\
\hline MMSE & $\begin{array}{c}12-30 \\
(n=113)\end{array}$ & $29(n=1)$ & $20(n=1)$ & & & \\
\hline MoCA & $\begin{array}{c}10-30 \\
(n=14)\end{array}$ & & & & & \\
\hline RASS & $\begin{array}{c}-1 \text { to } 1 \\
(n=114)\end{array}$ & & & & & \\
\hline DI & $\begin{array}{c}0-7 \\
(n=113)\end{array}$ & $0-3(n=9)$ & $0-4(n=5)$ & $0(n=2)$ & $0-2(n=2)$ & $2(n=1)$ \\
\hline DVT & $\begin{array}{l}81-103 \\
(n=27)\end{array}$ & $\begin{array}{l}86-101 \\
(n=12)\end{array}$ & $\begin{array}{c}85-103 \\
(n=6)\end{array}$ & $\begin{array}{l}92-101 \\
(n=3)\end{array}$ & $\begin{array}{l}95-100 \\
(n=3)\end{array}$ & \\
\hline CRT RT (sec) & $\begin{array}{c}0.78-11.68 \mathrm{~s} \\
(n=93)\end{array}$ & & & & & \\
\hline CRT Acc (\%) & $\begin{array}{c}40-95 \\
(n=93)\end{array}$ & & & & & \\
\hline Serious Game Median RT (s) & $\begin{array}{l}0.62-4.50 \\
(n=114)\end{array}$ & $\begin{array}{c}0.63-1.91 \\
(n=47)\end{array}$ & $\begin{array}{c}0.59-1.10 \\
(n=23)\end{array}$ & $\begin{array}{c}0.50-1.37 \\
(n=16)\end{array}$ & $\begin{array}{c}0.76-0.86 \\
(n=2)\end{array}$ & $0.82(n=1)$ \\
\hline Serious Game Target Offset (px) & $\begin{array}{c}243.50-449.00 \\
(n=114)\end{array}$ & $\begin{array}{c}218.00-409.00 \\
(n=47)\end{array}$ & $\begin{array}{c}202.00-365.50 \\
(n=23)\end{array}$ & $\begin{array}{c}162.00-339.00 \\
(n=16)\end{array}$ & $\begin{array}{c}306.50-331.00 \\
(n=2)\end{array}$ & $306.00(n=1)$ \\
\hline
\end{tabular}

TABLE 3 | Relationships between sessions on serious game median RT, was determined using two-tailed Pearson's $r$ correlations.

\begin{tabular}{lcccc}
\hline & $\begin{array}{c}\text { Initial } \\
\text { enrolment }\end{array}$ & $\begin{array}{c}\text { Follow } \\
\text { up 1 }\end{array}$ & $\begin{array}{c}\text { Follow } \\
\text { up 2 }\end{array}$ & $\begin{array}{c}\text { Follow } \\
\text { up 3 }\end{array}$ \\
\hline Initial enrolment & 1 & $\begin{array}{c}0.776^{* *} \\
p<0.001\end{array}$ & $\begin{array}{c}0.594^{* *} \\
p=0.003\end{array}$ & $\begin{array}{c}0.862^{* *} \\
p<0.001 \\
n=15\end{array}$ \\
Follow up 1 & $n=47$ & $n=23$ & $\begin{array}{c}n=1 \\
\end{array}$ \\
& 1 & $0.821^{* *}$ & $0.821^{* *}$ \\
Follow up 2 & & $p<0.001$ & $p=0.001$ \\
& & $n=19$ & $n=13$ \\
Follow up 3 & & 1 & $0.560^{*}$ \\
& & & & $n=0.037$ \\
& & & & $n=14$ \\
\end{tabular}

Shaded gray areas highlight adjacent sessions. ${ }^{*} p<0.05,{ }^{* *} p<0.01$.

offset values were not observed for the other two comparisons. A corresponding Wilcoxon signed-rank test showed that game target offset between initial enrolment and follow up session 1 was significantly different $(Z=-4.441, p<0.001)$. Participants had smaller target offset values in the first follow up sessions compared to initial enrolment. As with the $t$-tests, the Wilcoxon signed-rank tests did not show significant differences in target offset between follow up sessions 1 and 2, nor between follow up sessions 2 and 3.

Figure 5 visualizes potential practice and fatigue effects across successive follow up sessions. The figure shows histograms of the median RT differences (within participants) between successive sessions. In the initial enrolment and first follow up session, one patient with a difference greater than $1 \mathrm{~s}$ (the difference was greater than $2.5 \mathrm{~s}$ for this patient, but the $1 \mathrm{~s}$ difference was used as the cut-off) was omitted from the histogram. The histograms were then scaled to be on the same $\mathrm{x}$-axis with the same time bin sizes (each time bin had a width of $50 \mathrm{~ms}$ ) and were lined up vertically to facilitate visual comparison. We assume that
TABLE 4 | Relationship between serious game median target offset between each determined using two-tailed Pearson's $r$ correlations.

\begin{tabular}{lcccc}
\hline & $\begin{array}{c}\text { Initial } \\
\text { enrolment }\end{array}$ & $\begin{array}{c}\text { Follow } \\
\text { up 1 }\end{array}$ & $\begin{array}{c}\text { Follow } \\
\text { up 2 }\end{array}$ & $\begin{array}{c}\text { Follow } \\
\text { up 3 }\end{array}$ \\
\hline Initial enrolment & 1 & $\begin{array}{c}0.742^{* *} \\
p<0.001 \\
n=47\end{array}$ & $\begin{array}{c}0.658^{* *} \\
p=0.001 \\
n=23\end{array}$ & $\begin{array}{c}0.265 \\
p=0.340 \\
n=15\end{array}$ \\
Follow up 1 & 1 & $0.806^{* *}$ & 0.325 \\
& & $p<0.001$ & $p=0.279$ \\
& & $n=19$ & $n=13$ \\
Follow up 2 & & 1 & 0.497 \\
& & & & 0.071 \\
Follow up 3 & & & $n=14$ \\
\hline
\end{tabular}

Shaded gray areas highlight adjacent sessions. ${ }^{*} p<0.05,{ }^{* *} p<0.01$.

patients with a difference in median RT that was greater than zero in the subsequent session were in poorer condition or were experiencing fatigue. In contrast, for patients with a difference less than zero, there was likely a practice effect, as they were speeding up in the subsequent session. Since the distribution of differences in median RT between adjacent sessions (within individuals) is reasonably well balanced around the no difference $(0 \mathrm{~s})$ point there is little evidence of a genuine learning effect in game RT performance. Instead, the reduction in game median RT in later sessions (as indicated by significant $t$-tests and Wilcoxon signed-rank tests) is likely due, in most part, to slower participants dropping out of the study either because they were treated more quickly or because they were less willing or able to participate in the later sessions.

\section{DISCUSSION}

The present findings demonstrate the test-retest reliability of our game-based screening tool with an elderly emergency population. 


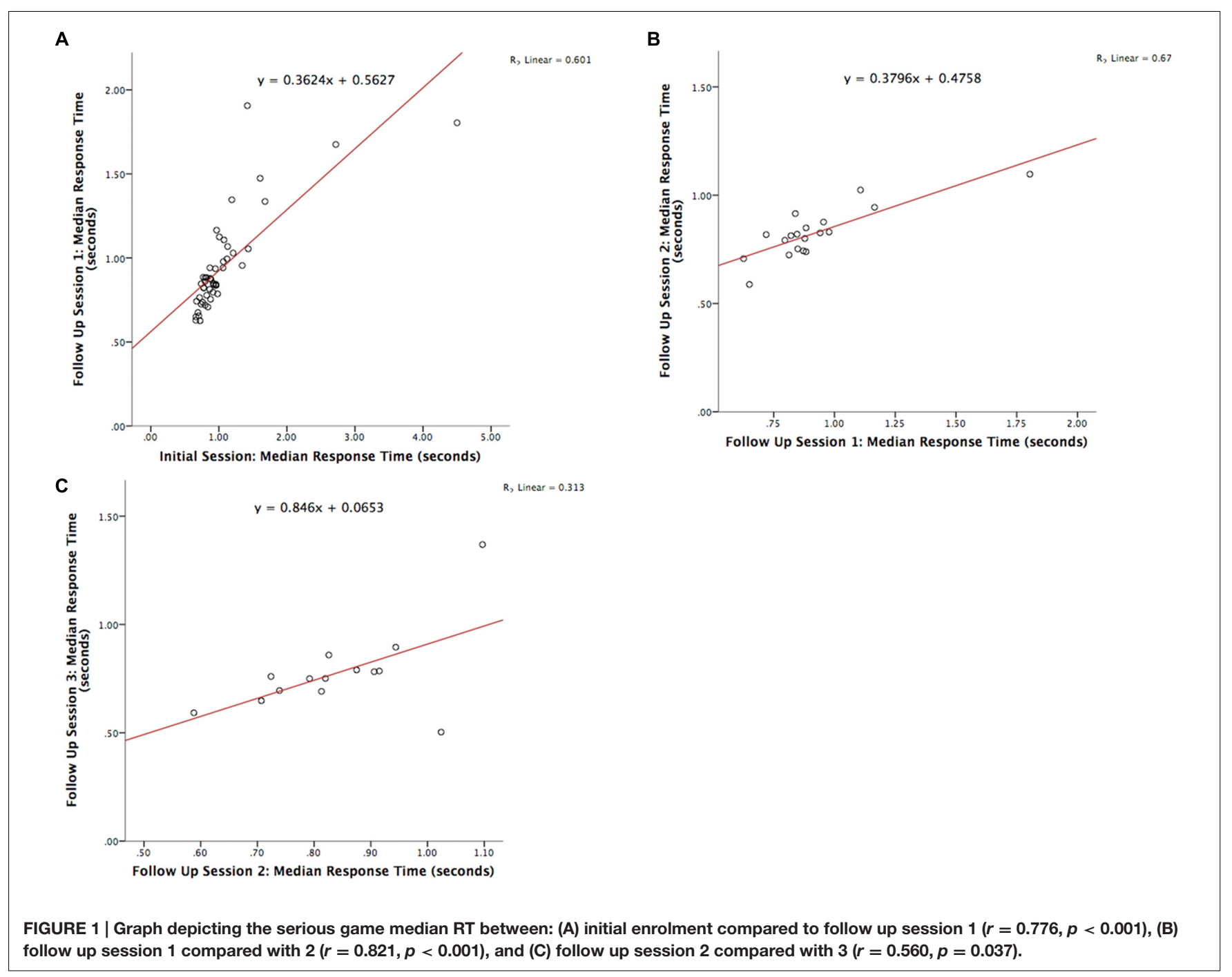

We observed strong relationships between all possible pairs of administrations of our serious game (initial enrolment, and follow up sessions 1 through 3 ) with $r$-values generally ranging between 0.56 and 0.82 for both median RT, and median target offset values (Tables 3 and 4). Similar Spearman's rho-values ranges were observed for game median $\mathrm{RT}$, and for median target offset (see Tables 5 and $\mathbf{6}$ ), respectively.

In the initial enrolment, we observed a wide range of MMSE (12-30) and MoCA (10-30) scores. Patients with possible dementia (MMSE scores below 24) (O'Connor et al., 1989) and MCI (MoCA scores below 23) (Luis et al., 2009) were still able to play our serious game. This suggests that people with cognitive impairments can use our game-based cognitive assessment.

The game median RT performance over sessions, within patients, was relatively consistent with the histograms of within patient RT differences between adjacent sessions (Figure 5) balanced around the zero difference value. Thus there is no evidence for the presence of a practice/learning effect.

We expected that many patients who were high performing would be assessed and discharged prior to their second assessment and that game performance would likely get worse in later sessions. However, this was not the case. Thus it appears that the sample of patients who remained in the ED over an extended period of time, remained CAM negative, and who were able to play the game, were comparatively fit. They had better game performance than the patients who dropped out of the study after the earlier sessions. With respect to the fact that within subjects there was no tendency for either speeding up or slowing down across sessions, it is possible that practice/learning effects (speeding up) precisely matched the fatigue effects (slowing down). However, a more parsimonious explanation might be that there were no practice or fatigue effects in this case, which would be a beneficial property of the game if it can be further verified in future research.

For cognitive assessment, the reliability of game RT is of most interest since game RT was found to correlate better (than game target offset) with other clinical assessments in the previous concurrent validity study (Tong et al., 2016). The test-retest reliability correlations obtained with game RT in this study were comparable with the test-retest correlations obtained by 
A



C



B

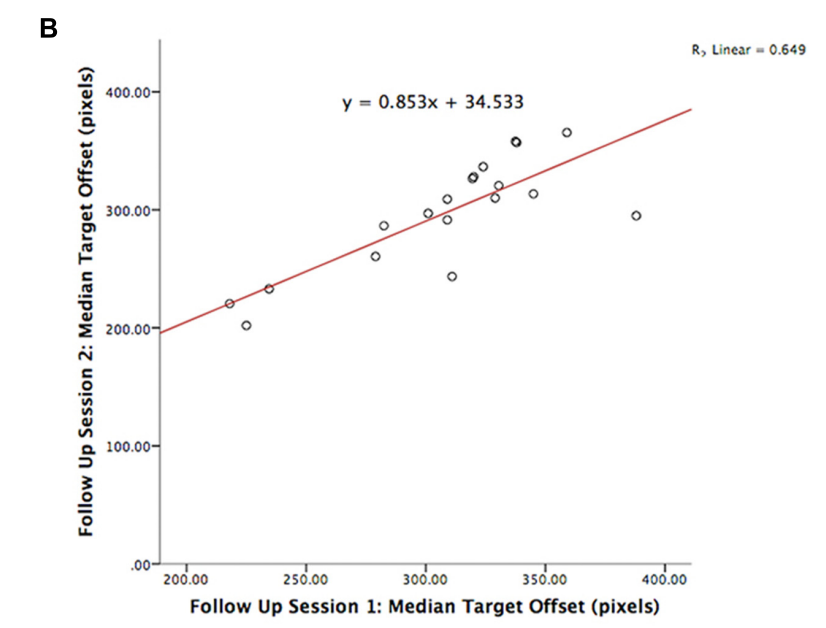

ollow Up Session 1: Median Target Offset (pixels)

, Linear $=0.247$

$R$, Linear $=0.552$

FIGURE 2 | Graph depicting the serious game median target offset between: (A) initial enrolment compared to follow up session 1 ( $r=0.742$, $p<0.001$ ), (B) follow up session 1 compared $2(r=0.806, p<0.001)$, and (C) follow up session 2 compared $3(r=0.497, p=0.071)$.

Tombaugh (2005) in his study of the test-retest reliability of the MMSE. However, in the present study follow up sessions were separated by 8 -h, whereas in Tombaugh's (2005) study the different time periods were separated by one or more years. In addition, the MoCA has been shown to have a high test-retest reliability of 0.92 over a period of around a month (Nasreddine et al., 2005), which is significantly longer than the 8-h separation between follow ups in the present study. In cases where short test-retest time intervals were used, test-retest reliability of the MMSE was reported to be much higher (between 0.80 and 0.95). There are likely a number of reasons why previous published values may tend to overestimate the value of the test-retest reliability of the MMSE, of which two are considered here. First, the MMSE studies generally selected people who were not assessed to be cognitively impaired (e.g., the participants would have had MMSE scores relatively close to the top end of the MMSE scale). This would have resulted in a compressed scale, making it more likely that scores within individuals would tend not to change (in contrast to game RT where the RTs may vary over many milliseconds). Second, since the MMSE items are identical and since the focus is on unimpaired individuals, scores will tend to stay the same or possibly improve due to practice effects and the ability to remember items on the test. In contrast to the MMSE, a patient cannot remember an exact sequence of events in the serious game that we used, since where and when targets appear was varying, and determined probabilistically. In the present study, we found little evidence of practice effects in game median RT performance, when time differences within individuals were assessed (Figure 5).

\section{Limitations and Future Work}

Our study focused on patients admitted to one hospital ED. It is possible that somewhat different findings might have been obtained in a different ED. In future studies, research should explore a more diverse patient population so as to improve the generalizability of the results. While we did not observe a strong learning (practice) effect in game performance between sessions, it is likely that there was an initial learning effect when patients first started using the game. We did not attempt to assess the initial learning effect in this study. Instead we allowed patients to do some initial practice with the game, with feedback and encouragement provided by the RAs, before the patient played 
TABLE 5 | Relationships between sessions on serious game median RT, as determined using two-tailed Spearman's rho correlations.

\begin{tabular}{lcccc}
\hline & $\begin{array}{c}\text { Initial } \\
\text { enrolment }\end{array}$ & $\begin{array}{c}\text { Follow } \\
\text { up 1 }\end{array}$ & $\begin{array}{c}\text { Follow } \\
\text { up 2 }\end{array}$ & $\begin{array}{c}\text { Follow } \\
\text { up 3 }\end{array}$ \\
\hline Initial enrolment & 1 & $\begin{array}{c}0.853^{* *} \\
p<0.001\end{array}$ & $\begin{array}{c}0.534^{* *} \\
p=0.009\end{array}$ & $\begin{array}{c}0.618^{* *} \\
p=0.014 \\
n=15\end{array}$ \\
Follow up 1 & $n=47$ & $n=23$ & $\begin{array}{c}n=158^{* *} \\
\text { Follow up 2 }\end{array}$ \\
& 1 & $0.741^{* *}$ & 0.580 .035 \\
& & $n=19$ & $n=13$ \\
& & & $0.560^{*}$ \\
& & & $p=0.037$ \\
& & & $n=14$
\end{tabular}

Follow up 3

1

Shaded gray areas highlight adjacent sessions. ${ }^{*} p<0.05,{ }^{* *} p<0.01$

TABLE 6 | Relationship between serious game median target offset, between each determined using two-tailed Spearman's rho correlations.

\begin{tabular}{lcccc}
\hline & $\begin{array}{c}\text { Initial } \\
\text { enrolment }\end{array}$ & $\begin{array}{c}\text { Follow } \\
\text { up 1 }\end{array}$ & $\begin{array}{c}\text { Follow } \\
\text { up 2 }\end{array}$ & $\begin{array}{c}\text { Follow } \\
\text { up 3 }\end{array}$ \\
\hline Initial enrolment & 1 & $\begin{array}{c}0.685^{* *} \\
p<0.001 \\
n=47\end{array}$ & $\begin{array}{c}0.451^{* *} \\
p=0.031 \\
n=23\end{array}$ & $\begin{array}{c}0.340 \\
p=0.216 \\
n=15\end{array}$ \\
Follow up 1 & 1 & $\begin{array}{c}0.777^{* *} \\
p<0.001\end{array}$ & $\begin{array}{c}0.484 \\
p=0.094 \\
n=13\end{array}$ \\
& & & $n=19$ & $0.741^{* *}$ \\
Follow up 2 & & & $p=0.002$ \\
& & & & $n=14$ \\
Follow up 3 & & & & 1
\end{tabular}

Shaded gray areas highlight adjacent sessions. ${ }^{*} p<0.05,{ }^{*} p<0.01$.

the game to make the assessment. In contrast to the apparent lack of practice effects for the serious game median RT, practice effects have been found in "standard" cognitive assessments such as the MoCA and MMSE.

While significant, the test-retest reliabilities observed here for the serious game are lower than the corresponding reliabilities reported for the MMSE (for short time periods of up to a few months). However, the game provides a much wider range of scores, since RT is measured in milliseconds and it is not possible to memorize the answers to questions as it may be for tests such as the MMSE.

We did not assess inter-rater reliability for the CAM (used to screen out delirious patients in this study). However, the CAM has been shown to have a high inter-rater reliability (Bhat and Rockwood, 2007). Moreover, we also observed a high rate of lossto-follow-up due to carrying out assessments every 8-h. Future studies should consider using shorter latencies between sessions (e.g., every 1-h) in order to improve the chances of detecting delirium.

\section{CONCLUSION}

We have demonstrated that our game-based screening tool is a reliable tool (in terms of test-retest reliability) for measuring
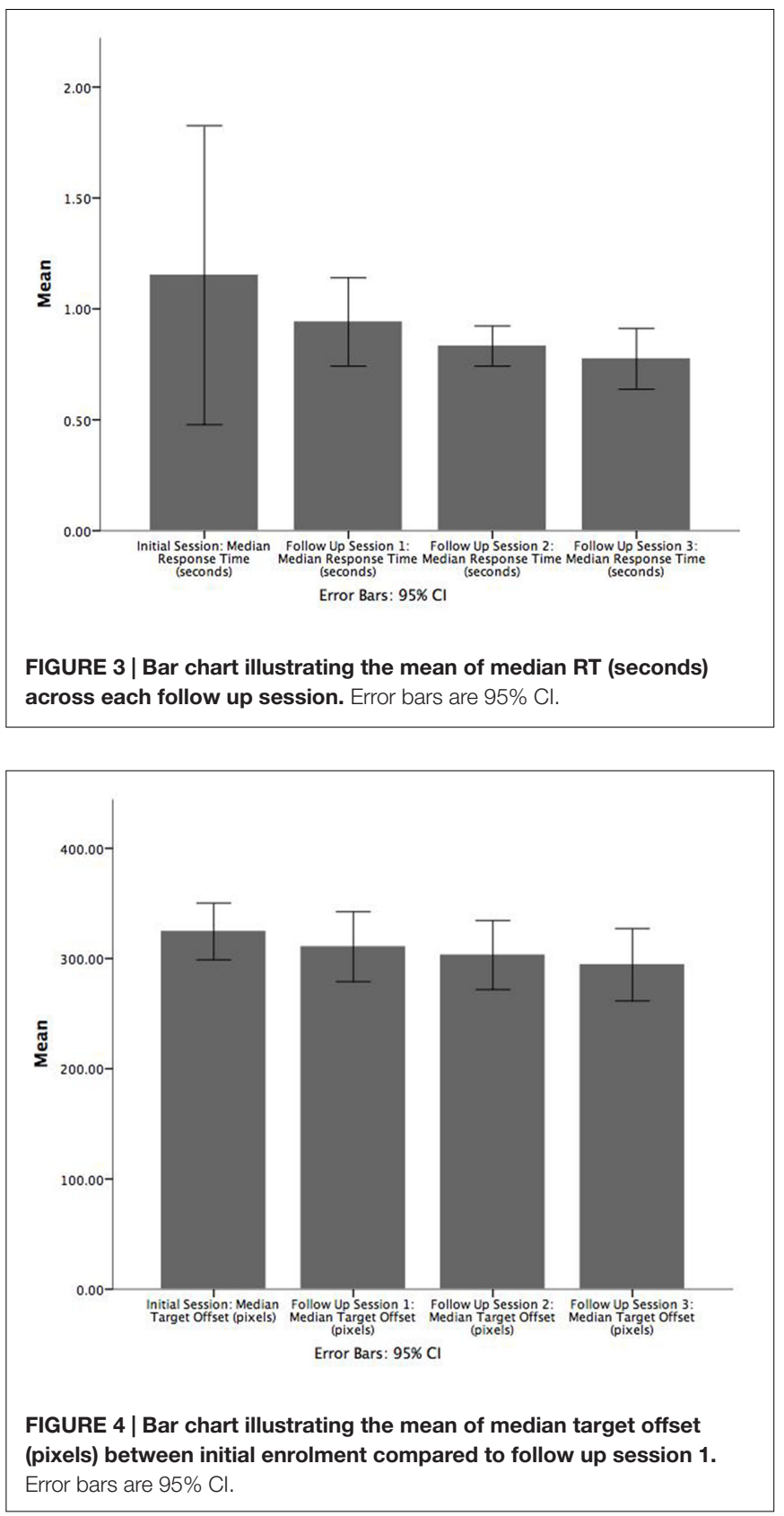

cognitive status, and that it can be used independently by patients in emergency care after a few minutes of training (at most). In related work we have also begun the process of validating our game-based scrreening tool by establishing that game median RT is significant correlated with standard clinical assessments such as the MMSE, and MoCA (Tong et al., 2016). Taken together, the present work and our previous study demonstrate that our serious game for cognitive assessment has good levels of both concurrent validity and test-retest reliability. The game is also usable, and can be self-administered by patients. While the game appears to have lower test-retest reliability than the MMSE, it does not seem to have practice effects if people are given a short 




FIGURE 5 | Histogram illustrating the median RT difference (seconds) between successive session. Each bin represents $0.05 \mathrm{~s}$.

amount of initial training, and it provides a wider range of scores and no opportunities for memorizing answers. While the serious game has yet to be fully validated for routine assessment of cognitive decline, this research opens the way to further explore self-administration by patients of a cognitive screening tool that is able to track their progress over time. In busy environments such as the ED, this type of serious game for self-administered cognitive assessment could, in the future, assist both healthcare providers and patients by providing critical information on a patient's cognitive status over time. The game should be a useful supplement to tests such as the MoCA, and MMSE in situations where it may be difficult or impractical to use those existing assessments.

\section{AUTHOR CONTRIBUTIONS}

TT designed and developed the game, carried out the statistical analyses, and wrote the first draft of the manuscript. JL designed the clinical study and supervised the data collection. MT assisted with the selection of clinical assessments used in the study and in the interpretation of the results. MC assisted in the research design and statistical analysis. All of the authors participated in the editing and revision of the manuscript.

\section{FUNDING}

MC and TT are supported by a grant from the AGE-WELL National Center of Excellence (WP 6.1). MCT is supported by a Clinician Scientist Award from the Department of Family and Community Medicine, University of Toronto. This research was funded by a Canadian Institutes of Health Research Catalyst Grant: eHealth Innovations (application number 316802).

\section{ACKNOWLEDGMENTS}

We would like to thank all volunteers who participated in our research studies. We would also like to thank Janahan Sandrakumar, Jacob Woldegabriel, and Joanna Yeung, for assisting with data collection. 


\section{REFERENCES}

Anguera, J. A., Boccanfuso, J., Rintoul, J. L., Al-Hashimi, O., Faraji, F., Janowich, J., et al. (2013). Video game training enhances cognitive control in older adults. Nature 501, 97-101. doi: 10.1038/nature12486

Anastasi, A. (1988). Psychological Testing: Anne, Anastasi: Amazon.com: Books, 6th Edn. New York, NY: Macmillan Publishing Company.

Bhat, R., and Rockwood, K. (2007). Delirium as a disorder of consciousness. J. Neurol. Neurosurg. Psychiatry 78, 1167-1170. doi: 10.1136/jnnp.2007.115998

Brouillette, R. M., Foil, H., Fontenot, S., Correro, A., Allen, R., Martin, C. K., et al. (2013). Feasibility, reliability, and validity of a smartphone based application for the assessment of cognitive function in the elderly. PLOS ONE 8:e65925. doi: 10.1371/journal.pone.0065925

Calamia, M., Markon, K., and Tranel, D. (2012). Scoring higher the second time around: meta-analyses of practice effects in neuropsychological assessment. Clin. Neuropsychol. 26, 543-570. doi: 10.1080/13854046.2012.680913

Cambridge Cognition Ltd (2014). Cantab. Cambridge: Cambridge Cognition Ltd.

Centers for Disease Control and Prevention (2013). The State of Aging and Health in America 2013. Atlanta: Centers for Disease Control and Prevention.

Charsky, D. (2010). From edutainment to serious games: a change in the use of game characteristics. Games Cult. 5, 177-198. doi: 10.1177/1555412009354727

Collerton, J., Collerton, D., Arai, Y., Barrass, K., Eccles, M., Jagger, C., et al. (2007). A comparison of computerized and pencil-and-paper tasks in assessing cognitive function in community-dwelling older people in the Newcastle 85+ Pilot Study. J. Am. Geriatr. Soc. 55, 1630-1635. doi: 10.1111/j.15325415.2007.01379.x

Cooley, S. A., Heaps, J. M., Bolzenius, J. D., Salminen, L. E., Baker, L. M., Scott, S. E., et al. (2015). Longitudinal change in performance on the montreal cognitive assessment in older adults. Clin. Neuropsychol. 29, 824-835. doi: 10.1080/13854046.2015.1087596

de Lange, E., Verhaak, P. F. M., and van der Meer, K. (2013). Prevalence, presentation and prognosis of delirium in older people in the population, at home and in long term care: a review. Int. J. Geriatr. Psychiatry 28, 127-134. doi: 10.1002 /gps.3814

Ely, E. W., Inouye, S. K., Bernard, G. R., Gordon, S., Francis, J., May, L., et al. (2001). Delirium in mechanically ventilated patients: validity and reliability of the confusion assessment method for the intensive care unit (CAM-ICU). JAMA 286, 2703-2710. doi: 10.1001/jama.286.21.2703

Field, A. (2013). Discovering Statistics using IBM SPSS Statistics, 4th Edn. Thousand Oaks, CA: SAGE Publications Ltd.

Folstein, M. F., Folstein, S. E., and McHugh, P. R. (1975). "Mini-mental state" a practical method for grading the cognitive state of patients for the clinician. J. Psychiatr. Res. 12, 189-198. doi: 10.1016/0022-3956(75)90026-6

Galasko, D., Abramson, I., Corey-Bloom, J., and Thal, L. J. (1993). Repeated exposure to the mini-mental state examination and the information-memoryconcentration test results in a practice effect in Alzheimer's disease. Neurology 43, 1559-1563. doi: 10.1212/WNL.43.8.1559

Gamberini, L., Alcaniz, M., Barresi, G., Fabregat, M., Ibanez, F., and Prontu, L. (2006). Cognition, technology and games for the elderly: an introduction to ELDERGAMES Project. PsychNology J. 4, 285-308.

Gershon, R. C., Wagster, M. V., Hendrie, H. C., Fox, N. A., Cook, K. F., and Nowinski, C. J. (2013). NIH toolbox for assessment of neurological and behavioral function. Neurology 80, S2-S6. doi: 10.1212/WNL. 0b013e3182872e5f

Glass, T. A., and Balfour, J. L. (2003). "Neighborhoods, aging, and functional limitations," in Neighbourhoods and Health, ed. B. L. Kawachi (New York, NY: Oxford University Press), 303-334.

Gualtieri, C. T., and Johnson, L. G. (2006). Reliability and validity of a computerized neurocognitive test battery, CNS vital signs. Arch. Clin. Neuropsychol. 21, 623-643. doi: 10.1016/j.acn.2006.05.007

Helkala, E.-L., Kivipelto, M., Hallikainen, M., Alhainen, K., Heinonen, H., Tuomilehto, J., et al. (2002). Usefulness of repeated presentation of mini-mental state examination as a diagnostic procedure-a population-based study. Acta Neurol. Scand. 106, 341-346. doi: 10.1034/j.1600-0404.2002.01315.x

Hustey, F., Meldon, S., and Palmer, R. (2000). Prevalence and documentation of impaired mental status in elderly emergency department patients. Acad. Emerg. Med. 7, 1166.
Hustey, F. M., and Meldon, S. W. (2002). The prevalence and documentation of impaired mental status in elderly emergency department patients. Ann. Emerg. Med. 39, 248-253. doi: 10.1067/mem.2002. 122057

Inouye, S. K. (1990). Clarifying confusion: the confusion assessment method. Ann. Intern. Med. 113, 941. doi: 10.7326/0003-4819-113-12-941

Inouye, S. K., van Dyck, C. H., Alessi, C. A., Balkin, S., Siegal, A. P., and Horwitz, R. I. (1990). Clarifying confusion: the confusion assessment method. A new method for detection of delirium. Ann. Intern. Med. 113, 941-948. doi: 10.7326/0003-4819-113-12-941

Inouye, S. K., Westendorp, R. G. J., and Saczynski, J. S. (2014). Delirium in elderly people. Lancet 383, 911-922. doi: 10.1016/S0140-6736(13)60688-1

Jacqmin-Gadda, H., Fabrigoule, C., Commenges, D., and Dartigues, J. F. (1997). A 5-year longitudinal study of the mini-mental state examination in normal aging. Am. J. Epidemiol. 145, 498-506. doi: 10.1093/oxfordjournals.aje. a009137

Kakuma, R., du Fort, G. G., Arsenault, L., Perrault, A., Platt, R. W., Monette, J., et al. (2003). Delirium in older emergency department patients discharged home: effect on survival. J. Am. Geriatr. Soc. 51, 443-450. doi: 10.1046/j.15325415.2003.51151.x

Kelland, D. (1996). The digit vigilance test: reliability, validity, and sensitivity to diazepam. Arch. Clin. Neuropsychol. 11, 339-344. doi: 10.1016/08876177(95)00032-1

LaMantia, M. A., Messina, F. C., Hobgood, C. D., Miller, D. K., Association, A. P., Siddiqi, N., et al. (2014). Screening for delirium in the emergency department: a systematic review. Ann. Emerg. Med. 63, 551.e-560.e. doi: 10.1016/j.annemergmed.2013.11.010

Luis, C. A., Keegan, A. P., and Mullan, M. (2009). Cross validation of the montreal cognitive assessment in community dwelling older adults residing in the southeastern US. Int. J. Geriatr. Psychiatry 24, 197-201. doi: 10.1002/gps. 2101

McCusker, J., Cole, M. G., Dendukuri, N., and Belzile, E. (2004). The delirium index, a measure of the severity of delirium: new findings on reliability, validity, and responsiveness. J. Am. Geriatr. Soc. 52, 1744-1749. doi: 10.1111/j.15325415.2004.52471.x

Meagher, D. J., Trzepacz, P., Khaw, K., Inouye, S., Rockwood, K., Cosway, S., et al. (2001). Delirium: optimising management. BMJ 322, 144-149. doi: 10.1136/bmj.322.7279.144

Nasreddine, Z. S., Phillips, N. A., Bédirian, V., Charbonneau, S., Whitehead, V., Collin, I., et al. (2005). The montreal cognitive assessment, MoCA: a brief screening tool for mild cognitive impairment. J. Am. Geriatr. Soc. 53, 695-699. doi: 10.1111/j.1532-5415.2005.53221.x

O'Connor, D. W., Pollitt, P. A., Hyde, J. B., Fellows, J. L., Miller, N. D., Brook, C. P. B., et al. (1989). The reliability and validity of the mini-mental state in a British community survey. J. Psychiatr. Res. 23, 87-96. doi: 10.1016/00223956(89)90021-6

Onoda, K., Hamano, T., Nabika, Y., Aoyama, A., Takayoshi, H., Nakagawa, T., et al. (2013). Validation of a new mass screening tool for cognitive impairment: cognitive assessment for dementia, iPad version. Clin. Interv. Aging 8, 353-360. doi: 10.2147/CIA.S42342

Petersen, R. C., Stevens, J. C., Ganguli, M., Tangalos, E. G., Cummings, J. L., and DeKosky, S. T. (2001). Practice parameter: early detection of dementia: mild cognitive impairment (an evidence-based review). Report of the quality standards subcommittee of the american academy of neurology. Neurology 56, 1133-1142. doi: 10.1212/WNL.56.9.1133

Pun, B. T., and Ely, E. W. (2007). The importance of diagnosing and managing ICU delirium. Chest 132, 624-636. doi: 10.1378/chest.06-1795

Rudra, A., Chatterjee, S., Kirtania, J., Sengupta, S., Moitra, G., Sirohia, S., et al. (2006). Postoperative delirium. Indian J. Crit. Care Med. 10, 235. doi: 10.4103/0972-5229.29842

Saxton, J., Morrow, L., Eschman, A., Archer, G., Luther, J., and Zuccolotto, A. (2009). Computer assessment of mild cognitive impairment. Postgrad. Med. 121, 177-185. doi: 10.3810/pgm.2009.03.1990

Sessler, C. N., Gosnell, M. S., Grap, M. J., Brophy, G. M., O’Neal, P. V., Keane, K. A., et al. (2002). The richmond agitation-sedation scale: validity and reliability in adult intensive care unit patients. Am. J. Respir. Crit. Care Med. 166, 1338-1344. doi: $10.1164 / \mathrm{rccm} .2107138$ 
Tierney, M. C., and Lermer, M. A. (2010). Computerized cognitive assessment in primary care to identify patients with suspected cognitive impairment. J. Alzheimer's Dis. 20, 823-832. doi: 10.3233/JAD-2010-09 1672

Tombaugh, T. N. (2005). Test-retest reliable coefficients and 5-year change scores for the MMSE and 3MS. Arch. Clin. Neuropsychol. 20, 485-503. doi: 10.1016/j.acn.2004.11.004

Tombaugh, T. N., and McIntyre, N. J. (1992). The mini-mental state examination: a comprehensive review. J. Am. Geriatr. Soc. 40, 922-935. doi: 10.1111/j.15325415.1992.tb01992.x

Tong, T., and Chignell, M. (2014). "Developing serious games for cognitive assessment: aligning game parameters with variations in capability," in Proceedings of the Second International Symposium of Chinese CHI on-Chinese CHI '14, (New York, NY: ACM Press), 70-79.

Tong, T., Chignell, M., Tierney, M. C., and Lee, J. (2016). A serious game for clinical assessment of cognitive status: validation study. JMIR Serious Games 4:e7. doi: 10.2196/games.5006

Wild, K., Howieson, D., Webbe, F., Seelye, A., and Kaye, J. (2008). Status of computerized cognitive testing in aging: a systematic review. Alzheimers. Dement. 4, 428-437. doi: 10.1016/j.jalz.2008.07.003

Wright, D. W., Goldstein, F. C., Kilgo, P., Brumfield, J. R., Ravichandran, T., Danielson, M. L., et al. (2010). Use of a novel technology for presenting screening measures to detect mild cognitive impairment in elderly patients. Int. J. Clin. Pract. 64, 1190-1197. doi: 10.1111/j.1742-1241.2009. 02324.x

Yechiam, E., Goodnight, J., Bates, J. E., Busemeyer, J. R., Dodge, K. A., Pettit, G. S., et al. (2006). A formal cognitive model of the go/no-go discrimination task: evaluation and implications. Psychol. Assess. 18, 239-249. doi: 10.1037/10403590.18.3.239

Zaslavsky, O., Thompson, H., and Demiris, G. (2012). The role of emerging information technologies in frailty assessment. Res. Gerontol. Nurs. 5, 216-228. doi: 10.3928/19404921-20120410-02

Zuchella, C., Sinforiani, E., Tassorelli, C., Cavallini, E., Tost-Pardell, D., Grau, S., et al. (2014). Serious games for screening pre-dementia conditions: from virtuality to reality? a pilot project. Funct. Neurol. 29, 153-158. doi: 10.11138/FNeur/2014.29.3.153

Conflict of Interest Statement: The authors declare that the research was conducted in the absence of any commercial or financial relationships that could be construed as a potential conflict of interest.

Copyright (c) 2016 Tong, Chignell, Tierney and Lee. This is an open-access article distributed under the terms of the Creative Commons Attribution License (CC BY).

The use, distribution or reproduction in other forums is permitted, provided the original author(s) or licensor are credited and that the original publication in this journal is cited, in accordance with accepted academic practice. No use, distribution or reproduction is permitted which does not comply with these terms. 